\title{
TOTAL-VARIATION REGULARIZATION WITH BOUND CONSTRAINTS
}

\author{
Rick Chartrand, Brendt Wohlberg \\ Los Alamos National Laboratory \\ \{rickc, brendt\}@lanl.gov
}

\begin{abstract}
We present a new algorithm for bound-constrained totalvariation (TV) regularization that in comparison with its predecessors is simple, fast, and flexible. We use a splitting approach to decouple TV minimization from enforcing the constraints. Consequently, existing TV solvers can be employed with minimal alteration. This also makes the approach straightforward to generalize to any situation where TV can be applied. We consider deblurring of images with Gaussian or salt-and-pepper noise, as well as Abel inversion of radiographs with Poisson noise.
\end{abstract}

Index Terms - Total variation, bound constraints, nonnegativity constraint, image deblurring, Abel inversion.

\section{INTRODUCTION}

Total-variation (TV) regularization [1] has been invaluable for image restoration and reconstruction, but the nonsmoothness of TV makes its implementation challenging. Numerous algorithms have been developed: primal-dual methods [2], iterative reweighting [3, 4], dual methods [5, 6], graph cuts [7], operator splitting [8], linear programming [9], second-order cone programming [10], and more.

TV was originally formulated [1] as a constrained optimization problem:

$$
\min _{u} \int_{\Omega}|\nabla u|, \text { subject to } \int_{\Omega}(u-f)^{2} \leq \sigma^{2} .
$$

Here, $f$ is a grayscale image defined on $\Omega \subset \mathbb{R}^{2}$, and $\sigma^{2}$ is the variance of Gaussian noise presumed to be present in $f$. However, it is generally regarded to be computationally simpler to solve an unconstrained formulation:

$$
\min _{u} \int_{\Omega}|\nabla u|+\frac{\lambda}{2} \int_{\Omega}(u-f)^{2} .
$$

For any value of $\sigma$, there is a corresponding value of $\lambda$ making (2) equivalent to (1), though it is not typically possible to determine the appropriate $\lambda$ a priori.

Further extensions of TV are obtained by considering a (typically linear) transformation $A$ :

$$
\min _{u} \int_{\Omega}|\nabla u|+\frac{\lambda}{2} \int_{\Omega}(A u-f)^{2} .
$$

Work supported by the LANL Inertial Confinement Fusion program.
The most common instance is when $A$ is a blurring operator, in which case solving (3) is TV-regularized deblurring of $f$. However, numerous other inverse processes have been similarly regularized, such as MRI [11] and CT [12] tomography, Abel inversion [13], and differentiation [14].

We can often include information about the values of the solution. The most common such constraint is that to be physically meaningful, the solution should be nonnegative. One approach is to simply impose this after solving (3), by clipping the solution. However, better results can be obtained by enforcing the constraint during the solution process itself:

$$
\min _{u} \int_{\Omega}|\nabla u|+\frac{\lambda}{2} \int_{\Omega}(A u-f)^{2}, \text { subject to } u \in[a, b],
$$

where $u \in[a, b]$ is meant pointwise (and similarly henceforth), and the interval may be unbounded. With $a=0$ and $b=\infty$, we have a nonnegativity constraint. Algorithms for solving (4) have been considered before, but in this work we present a new approach that is conceptually simpler and computationally faster.

\section{SPLITTING ALGORITHM FOR BOUND-CONSTRAINED TV}

Our algorithm uses a splitting approach, which decouples the tasks of minimizing TV from that of enforcing the constraint. We enforce the constraint on a new variable, $w$, which we link to $u$ initially by means of a trivial equality constraint:

$$
\begin{aligned}
\min _{u, w} \int_{\Omega}|\nabla u|+\frac{\lambda}{2} \int_{\Omega}(A u-f)^{2}, \\
\text { subject to } u=w \text { and } w \in[a, b] .
\end{aligned}
$$

However, we now relax the constraint $u=w$, instead penalizing the discrepancy between $u$ and $w$ :

$$
\begin{array}{r}
\min _{u, w} \int_{\Omega}|\nabla u|+\frac{\lambda}{2} \int_{\Omega}(A u-f)^{2}+\frac{\beta}{2} \int_{\Omega}(u-w)^{2}, \\
\text { subject to } w \in[a, b],
\end{array}
$$

where $\beta$ is a new parameter. We proceed by alternate minimization, fixing one variable and solving for the other. 
We first fix the iterate $w$, and solve for $u$, leaving the unconstrained problem

$$
\min _{u} \int_{\Omega}|\nabla u|+\frac{\lambda}{2} \int_{\Omega}(A u-f)^{2}+\frac{\beta}{2} \int_{\Omega}(u-w)^{2} .
$$

The corresponding Euler-Lagrange equation is

$$
-\nabla \cdot \frac{\nabla u}{|\nabla u|}+\lambda A^{T}(A u-f)+\beta(u-w) .
$$

This differs from the Euler-Lagrange equation for (3) only in the presence of the final term, which does not appreciably alter the difficulty of its solution. We solve (8) by using the linearization obtained by substituting the previous iterate $u^{(n)}$ for $u$ in the denominator of the first term. This is equivalent to using $u=u^{(n)}$ to generate a quadratic approximation to (7); see [4] for details of our iteratively-reweighted norm (IRN) approach. Convergence for the cases we consider in this paper has been established $[15,16]$. Most TV-regularization solvers can easily be modified to solve this subproblem.

Now we fix $u$ and solve for $w$ :

$$
\min _{w} \frac{\beta}{2} \int_{\Omega}(u-w)^{2} \text {, subject to } w \in[a, b] .
$$

We have a trivial, separable, quadratic optimization problem, with a simple solution: at each point $x, w(x)$ is the closest element of $[a, b]$ to $u(x)$ (hence $a, b$, or $u(x)$ itself). Note that it would be equally simple to use a separate interval at each pixel, but will we not pursue this here.

An additional consideration is that we wish the constraint $w=u$ to hold at convergence. For this, we use a method of multipliers approach (cf. the split Bregman approach of [17]). We incorporate a Lagrange multiplier $\nu$ :

$$
\begin{array}{r}
\min _{u, w} \int_{\Omega}|\nabla u|+\frac{\lambda}{2} \int_{\Omega}(A u-f)^{2}+\frac{\beta}{2} \int_{\Omega}(u-w-\nu)^{2}, \\
\text { subject to } w \in[a, b],
\end{array}
$$

which we update each iteration: $\nu^{(n+1)}=\nu^{(n)}+w^{(n+1)}-$ $u^{(n+1)}$. Standard method of multipliers theory [18] guarantees that $w^{(n)}-u^{(n)} \rightarrow 0$ as desired. Note that the inclusion of $\nu$ does not increase the difficulty of either of the subproblems, serving only to additively modify $w$ when solving for $u$, and vice versa.

Since the only nontrivial computation, that of solving for $u$, reduces to a slight modification of a standard TV problem, one can take advantage of previous instances where (3) has been generalized. We consider two such cases in this paper, in addition to TV-regularized deblurring: $L^{1}$-TV for deblurring images corrupted by salt-and-pepper noise [19, 20], and Abel inversion of images corrupted by Poisson noise $[13,16]$.

\section{EXPERIMENTS AND GENERALIZATIONS}

We first consider a nonnegatively-constrained TV deblurring problem. We blur a $128 \times 128$ satellite image with a $9 \times 9$

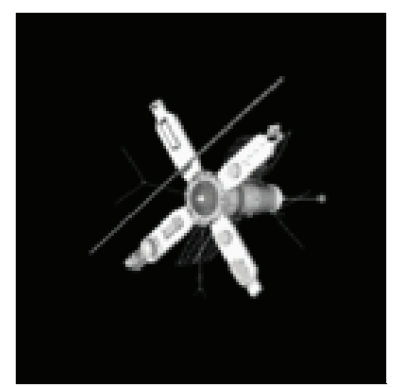

(a) Satellite image

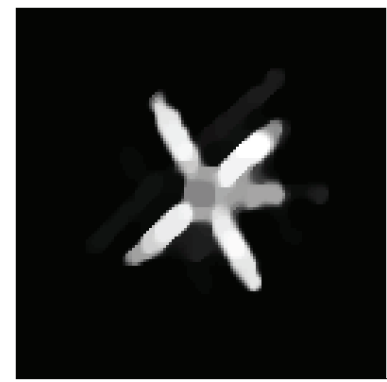

(c) NNCGM, SNR $13.2 \mathrm{~dB}, 89 \mathrm{~s}$

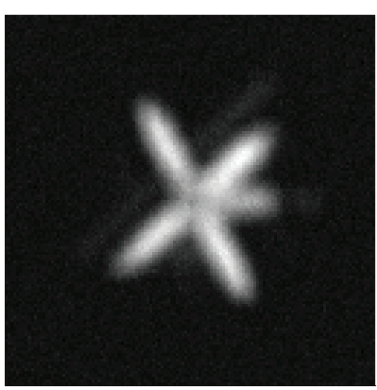

(b) Blurry, noisy (SNR $5.8 \mathrm{~dB}$ )

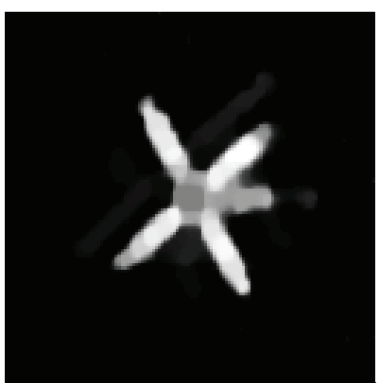

(d) IRN, SNR $13.2 \mathrm{~dB}, 36 \mathrm{~s}$
Fig. 1. Our reconstruction is similar to that of NNCGM [21], but in much less time.

Gaussian kernel $(\sigma=20)$, then add Gaussian noise, giving a resulting image having an SNR of $5.8 \mathrm{~dB}$. We minimize (10), using 10 iterations of alternation and Lagrange multiplier update. We compare our result with the primal-dual algorithm (called NNCGM) of Krishnan, Lin, and Yip [21], using their code. We obtain similar restorations, but ours runs almost 3 times faster. Our approach also has the flexibility to incorporate not only more general constraints, but also alternate noise models, with little alteration.

We now consider another TV deblurring problem, but with salt-and-pepper noise. We replace the $L^{2}$-norm data fidelity term in (3) with an $L^{1}$ norm instead, which is known to give substantially better results $[19,20]$ :

$$
\begin{array}{r}
\min _{u, w} \int_{\Omega}|\nabla u|+\frac{\lambda}{2} \int_{\Omega}|A u-f|+\frac{\beta}{2} \int_{\Omega}(u-w-\nu)^{2}, \\
\text { subject to } w \in[a, b] .
\end{array}
$$

The subproblem of solving for $u$ for fixed $w$ is a trivial modification of a standard $L^{1}$-TV regularization. The same approach of iteratively approximating (11) with a quadratic problem works well here too, noting that now the data fidelity term is approximated as well. The equation we solve for $u=u^{(n+1)}$ becomes (see [20] for further details):

$$
-\nabla \cdot \frac{\nabla u}{\left|\nabla u^{(n)}\right|}+\lambda A^{T} \frac{A u-f}{\left|A u^{(n)}-f\right|}+\beta\left(u-w^{(n)}-\nu^{(n)}\right) .
$$

We apply this approach to the $256 \times 256$ cameraman image (Figure 2(a)). We blur with a $7 \times 7$ Gaussian kernel $(\sigma=$ 


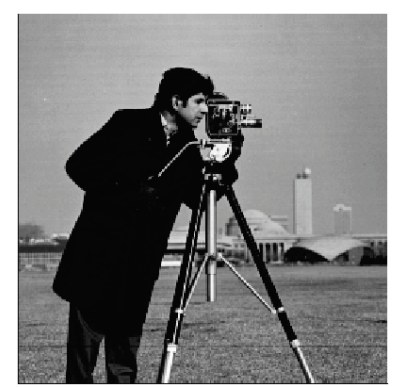

(a) Cameraman image

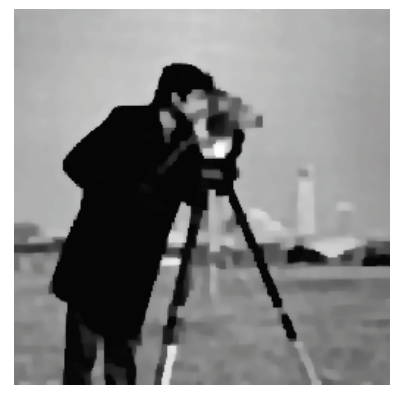

(c) LAD, SNR $11.1 \mathrm{~dB}, 239 \mathrm{~s}$

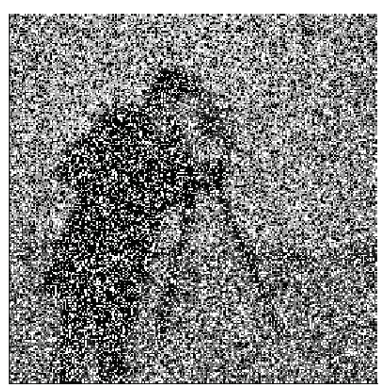

(b) Blurry, $60 \%$ pixels corrupted

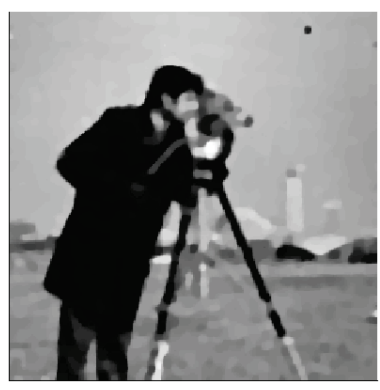

(d) IRN, SNR $11.1 \mathrm{~dB}, 85 \mathrm{~s}$ (SNR -0.4 dB)

Fig. 2. Our reconstruction is similar to that of LAD [9], but in much less time.

5 ), and then corrupt $60 \%$ of the pixels with salt-and-pepper noise. We compare with the linear programming, interiorpoint method (called LAD) of Fu, $\mathrm{Ng}$, Nikolova, and Barlow [9], as presented in [22] on a similar but not identical computer. We obtain similar results in less time, especially for heavily corrupted images, despite the LAD implementation containing some $\mathrm{C}$ code, while ours is entirely Matlab based. The LAD algorithm is limited to anisotropic TV $\left(\int\left(\left|u_{x}\right|+\right.\right.$ $\left.\left|u_{y}\right|\right)$, as opposed to $\int \sqrt{u_{x}^{2}+u_{y}^{2}}$ ), as the usual isotropic TV would require a second-order cone program. Our algorithm is also easily extended to data fidelity terms not having a linear programming formulation, such as the Poisson noise case we consider next.

It should be noted that in [8], Wang et al. consider a more aggressive splitting, decoupling the nonsmooth minimization task from the differentiation and measurement operators. Their $L^{1}$-TV algorithm runs much faster than ours in the previous example (17 s). This is without a nonnegativity constraint, but our constraint splitting can be incorporated into their approach. However, their efficiency relies on the fact that when $A$ is a blurring operator, $A^{T} A$ can be diagonalized by a Fourier transform. In contrast, our approach can easily be applied to operators without a useful Fourier representation, such as the Abel transform [13] we now consider.

We examine the task of inverting radiographs of an axisymmetric object. A reasonable approximation is that the negative-log of the radiograph is the Abel transform of a ra- dial half-slice of the object (this being sufficient to determine the whole object, due to the symmetry). We thus may proceed with (10), with $A$ being the Abel transform, and $f$ the negative-log radiograph. However, the noise in the radiograph is Poisson distributed, and in particular is signal dependent. Using a uniform regularization strength across the image will result in underregularization where the transmission is high, and/or overregularization where the transmission is low.

It is well known that a more appropriate data-fidelity term for Poisson noise is $\int(A u-f \log (A u))$. The iterativereweighting framework was extended to this case in [16]. The equation to be solved for $u$ becomes

$$
-\nabla \cdot \frac{\nabla u}{\left|\nabla u^{n}\right|}+\lambda A^{T} \frac{A u-f}{\left|A u^{n}\right|}+\beta\left(u-w^{n}-\nu^{n}\right) .
$$

The denominator of the second term serves to automatically rescale the regularization strength, by decreasing the "effective" $\lambda$ when the current estimate of $A u$ is large, and decreasing it when $A u$ is small. This is as desired when $f$ has Poisson noise, but in this case it is $e^{-f}$ that has Poisson noise, which inverts the usual relation to signal strength and noise variance. Hence we replace the denominator of the second term with a more appropriate scaling:

$$
-\nabla \cdot \frac{\nabla u}{\left|\nabla u^{(n)}\right|}+\lambda A^{T} \frac{A u-f}{e^{-A u^{(n)}}}+\beta\left(u-w^{(n)}-\nu^{(n)}\right) .
$$

We apply this approach to a simulated radiograph of a National Ignition Facility cryogenic target implosion ${ }^{1}$. The results are in Figure 3. We see that minimizing with respect to the nonnegativity constraint allows interior features to be preserved better than clipping. Using the Poisson noise approach of (13) results in better recovery of high density values (where transmission, hence noise, is lower), yet better noise removal in low density regions (where transmission, hence noise, is higher). We note further when a priori knowledge of the maximum density is available, using an upper bound constraint can further improve results. This is particularly so in the case of Abel inversion, since values near the axis are sensitive to the inaccuracies further from the axis. However, such knowledge was not available in this case.

\section{CONCLUSIONS}

Our proposed algorithm for bound-constrained TV regularization provides a useful balance between flexibility and computational efficiency; it is simpler to implement and faster than most competing algorithms $[9,21]$, and capable of solving a wider range of problems than one algorithm which could outperform it computationally [8, with our splitting added].

\footnotetext{
${ }^{1}$ Simulation by Charles Cerjan, LLNL.
} 


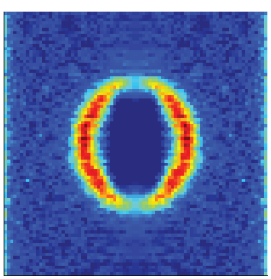

(a) TV-regularized Abel inverse, clipped at 0

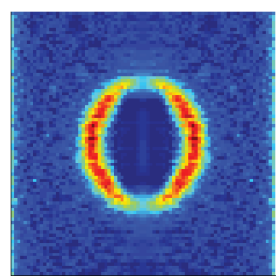
TV-regularized Abel inverse

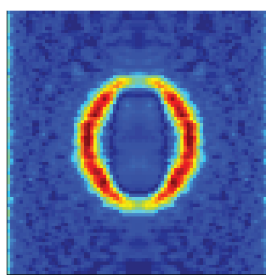

(c) constrained TV-regularized Abel inverse, with Poisson noise model
Fig. 3. Comparison of three TV-regularized Abel inversions. Using a nonnegativity constraint (b) gives more detail in the interior. The Poisson noise model (c) results in better noise removal, without high-density features being diminished.

\section{REFERENCES}

[1] L. Rudin, S. Osher, and E. Fatemi, "Nonlinear total variation based noise removal algorithms," Physica D, vol. 60, pp. 259-268, 1992.

[2] T. F. Chan, G. H. Golub, and P. Mulet, "A nonlinear primal-dual method for total variation-based image restoration," SIAM J. Sci. Comput., vol. 20, pp. 19641977, 1999.

[3] C. R. Vogel and M. E. Oman, "Iterative methods for total variation denoising," SIAM J. Sci. Comput., vol. 17, no. 1, pp. 227-238, 1996.

[4] P. Rodríguez and B. Wohlberg, "Efficient minimization method for a generalized total variation functional," IEEE Trans. Image Process., vol. 18, pp. 322-332, 2009.

[5] J. L. Carter, Dual Methods for Total Variation-Based Image Restoration. PhD thesis, University of California, Los Angeles, 2001.

[6] A. Chambolle, "An algorithm for total variation minimization and applications," J. Math. Imaging Vision, vol. 20, pp. 89-97, 2004.

[7] J. Darbon and M. Sigelle, "Image restoration with discrete constrained total variation part I: Fast and exact optimization," J. Math. Imaging Vision, vol. 26, no. 3, pp. 261-276, 2006.

[8] Y. Wang, J. Yang, W. Yin, and Y. Zhang, "A new alternating minimization algorithm for total variation image reconstruction," SIAM J. Imaging Sci., vol. 1, pp. 248272, 2008.

[9] H. Fu, M. K. Ng, M. Nikolova, and J. L. Barlow, "Efficient minimization methods of mixed 12-11 and 11-11 norms for image restoration," SIAM J. Sci. Comput., vol. 27, pp. 1881-1902, 2006.
[10] D. Goldfarb and W. Yin, "Second-order cone programming methods for total variation based image restoration,” SIAM J. Sci. Comput., vol. 27, pp. 622-645, 2005.

[11] M. Lustig, D. Donoho, and J. M. Pauly, "Sparse MRI: The application of compressed sensing for rapid MR imaging," Magn. Reson. Med., vol. 58, pp. 1182-1195, 2007.

[12] E. Y. Sidky, C. M. Kao, and X. C. Pan, "Accurate image reconstruction from few-views and limited-angle data in divergent-beam CT," J. X-ray Sci. Technol., vol. 14, pp. 119-139, 2006.

[13] T. J. Asaki, R. Chartrand, K. R. Vixie, and B. Wohlberg, "Abel inversion using total-variation regularization," Inverse Problems, vol. 21, pp. 1895-1903, 2005.

[14] R. Chartrand, "Numerical differentiation of noisy, nonsmooth data." preprint, 2007.

[15] T. F. Chan and P. Mulet, "On the convergence of the lagged diffusivity fixed point method in total variation image restoration," SIAM J. Numer. Anal., vol. 36, pp. 354-367, 1999.

[16] R. Chartrand and V. Staneva, "Total variation regularisation of images corrupted by non-Gaussian noise using a quasi-Newton method," IET Image Process., vol. 2, pp. 295-303, 2008.

[17] T. Goldstein and S. Osher, "The split Bregman method for L1 regularized problems," SIAM J. Imaging Sci., vol. 2, pp. 323-343, 2009.

[18] D. P. Bertsekas, Nonlinear Programming. Belmont, Massachusetts: Athena Scientific, 1999.

[19] M. Nikolova, "Minimizers of cost-functions involving nonsmooth data-fidelity terms. applications to the processing of outliers," SIAM J. Numer. Anal., vol. 40, pp. 965-994, 2002.

[20] B. Wohlberg and P. Rodríguez, "An $l^{1}$-TV algorithm for deconvolution with salt and pepper noise," in IEEE International Conference on Acoustics, Speech, and Signal Processing, pp. 1257-1260, 2009.

[21] D. Krishnan, P. Lin, and A. M. Yip, "A primal-dual active-set method for non-negativity constrained total variation deblurring problems," IEEE Trans. Image Process., vol. 16, pp. 2766-2777, 2007.

[22] J. Yang, Y. Zhang, and W. Yin, "An efficient TVL1 algorithm for deblurring multichannel images corrupted by impulsive noise," SIAM J. Sci. Comput., vol. 31, pp. 2842-2865, 2009. 